\title{
The concerns of the young protesters are justified
}

\author{
A statement by Scientists for Future concerning \\ the protests for more climate protection
}

\begin{abstract}
In March 2019, German-speaking scientists and scholars calling themselves Scientists for Future, published a statement in support of the youth protesters in Germany, Austria, and Switzerland (Fridays for Future, Klimastreik/Climate Strike), verifying the scientific evidence that the youth protestors refer to. In this article, they provide the full text of the statement, including the list of supporting facts (in both English and German) as well as an analysis of the results and impacts of the statement. Furthermore, they reflect on the challenges for scientists and scholars who feel a dual responsibility: on the one hand, to remain independent and politically neutral, and, on the other hand, to inform and warn societies of the dangers that lie ahead.
\end{abstract}

Gregor Hagedorn, Thomas Loew, Sonia I. Seneviratne, Wolfgang Lucht, Marie-Luise Beck, Janina Hesse, Reto Knutti, Volker Quaschning, Jan-Hendrik Schleimer, Linus Mattauch, Christian Breyer, Heike Hübener, Gottfried Kirchengast, Alice Chodura, Jens Clausen, Felix Creutzig, Marianne Darbi, Claus-Heinrich Daub, Felix Ekardt, Maja Göpel, Judith N. Hardt, Julia Hertin, Thomas Hickler, Arnulf Köhncke, Stephan Köster, Julia Krohmer, Helga Kromp-Kolb, Reinhold Leinfelder, Linda Mederake, Michael Neuhaus, Stefan Rahmstorf, Christine Schmidt, Christoph Schneider, Gerhard Schneider, Ralf Seppelt, Uli Spindler, Marco Springmann, Katharina Staab, Thomas F. Stocker, Karl Steininger, Eckart von Hirschhausen, Susanne Winter, Martin Wittau, Josef Zens

The concerns of the young protesters are justified. A statement by Scientists for Future concerning the protests for more climate protection | GAIA 28/2 (2019): 79-87

Keywords: biodiversity, climate action, Climate Strike, Fridays for Future, science-policy interface, sustainability crisis, youth movement

$A^{\prime}$ t present, many young people are demonstrating persistently for climate protection and the preservation of our natural resources. As scientists and scholars, and based on robust scientific evidence, we declare: these concerns are justified and supported by the best available science. The current measures for protecting the climate, biodiversity, and forest, marine, and soil resources, are far from sufficient.

The Paris Agreement of 2015 (UNFCCC 2015) obliges countries under international law to keep global warming well below $2{ }^{\circ} \mathrm{C}$. In addition, all countries have promised efforts to limit global warming to $1.5^{\circ} \mathrm{C}$. It is critical to immediately begin reducing net $\mathrm{CO}_{2}$ emissions and to eliminate them to zero worldwide between 2040 and 2050 at the latest (IPCC 2018). A more rapid reduction would increase the probability of not exceeding the $1.5^{\circ} \mathrm{C}$ limit. The use of coal should be nearly ended by 2030 , while the burning of oil and natural gas should be reduced simultaneously until all fossil fuels have been replaced by climate-neutral energy sources. Considering global climate justice, Europe must achieve this transition more quickly (IPCC 2018, Global Carbon Project 2018). While the need for participation and discussion remains, action must

Dr. Gregor Hagedorn | Berlin | Germany | g.m.hagedorn@gmail.com | orcid.org/0000-0001-7023-7386

(C) 2019 G. Hagedorn et al.; licensee oekom verlag. This Open Access article is published under the terms of the Creative Commons Attribution License CCBY 4.0 (http://creativecommons.org/licenses/by/4.0). https://doi.org/10.14512/gaia.28.2.3

Submitted May 4, 2019; revised version accepted May 10, 2019 be taken now. Discussion and action are not mutually exclusive. Many social and technological innovations already exist which can maintain quality of life and improve human well-being without destroying our natural resources (e.g., Klima-Allianz Deutschland 2018, WBGU 2011).

In all German-speaking countries, neither the necessary scale nor speed of change are being achieved in the restructuring of the energy, food, agriculture, resource, and mobility sectors. Germany will fail to meet the climate protection targets it has set itself for 2020 (UBA 2019), and the achievement of the goals of the German Sustainability Strategy for 2030 is at high risk (German Council for Sustainable Development 2018, SRU 2018). Moreover, there is still a lack of an effective climate protection law. Austria has set itself goals in its climate and energy strategy that do not in any way do justice to the Paris Agreement (CCCA 2018, Wegener Center für Klima und Globalen Wandel 2018, Schleicher and Kirchengast 2019) and even for this purpose, neither the necessary measures nor the financial means are provided (CCCA 2018). At the same time, soil degradation and surface coverage per person and year in Austria are the highest in Europe (UBA 2018). Switzerland has reduced its greenhouse gas emissions only slightly since 1990; at the same time, emissions caused abroad have increased considerably (BAFU 2018). In the first parliamentary debate on the total revision of the $\mathrm{CO}_{2} \mathrm{Act}$, the lower house proposed to abolish domestic reduction targets and to offset Swiss emissions abroad. In effect, the law has failed for the time being (Schweizer Parlament 2018). 


\section{BOX: Some important facts}

1. The global mean temperature has already risen by $1^{\circ} \mathrm{C}$ (relative to 1850 to 1900) (IPCC 2013, 2018). Half of the rise has occurred during the last 30 years (NASA 2018, IPCC 2014).

2. The years $2015,2016,2017$, and 2018 were, globally, the warmest years in the modern record (NASA 2019).

3. The temperature rise is almost entirely due to human-made greenhouse gas emissions (U.S. Global Change Research Program 2017, IPCC 2013 , 2014).

4. Already the current temperature rise increases the probability of extreme weather conditions in several regions of the globe, such as strong precipitation and heatwaves, leading to elevated rates of regional droughts, floods and forest fires (e.g., IPCC 2012, 2013, 2018, National Academies of Sciences, Engineering, and Medicine 2016).

5. Global warming is a risk factor for human health (Watts et al. 2015, 2018) Besides the above-mentioned direct consequences, its indirect consequences include the lack of food security and the spread of pathogens and disease carriers.

6. If humanity fails to limit global warming to $1.5^{\circ} \mathrm{C}$, as envisaged by the Paris Agreement, additional severe consequences must be expected for humanity and nature at large in many parts of the world (IPCC 2018).

7. In order to restrict warming to the $1.5^{\circ} \mathrm{C}$ limit with high probability, net emissions of greenhouse gases (in particular $\mathrm{CO}_{2}$ ) must be swiftly reduced and must, at the global level, reach zero within the next 20 to 30 years (IPCC 2013, 2018).

8. Instead, $\mathrm{CO}_{2}$-emissions continue to rise. Given the policy proposals currently on the table, global warming is likely to cross $3^{\circ} \mathrm{C}$ by the end of the century and will increase afterwards due to continued emissions and positive feedback dynamics (Climate Action Tracker 2018).

9. Based on current emissions, the remaining $\mathrm{CO}_{2}$-budget left for reaching the $1.5^{\circ} \mathrm{C}$ goal will last for about ten years. For the $2{ }^{\circ} \mathrm{C}$ goal, the budget is likely to last for about 25 to 30 years (MCC 2018, IPCC 2018).

10. Afterwards, humanity lives on a " $\mathrm{CO}_{2}$-overdraft-loan": any emitted greenhouse gases have to be removed later from the atmosphere with tremendous efforts (e.g., Rogelj et al. 2018, Gasser et al. 2015). Today's young people are already supposed to pay off this loan. If this fails, the following generations will suffer from the severe consequences of global warming.

11. Rising temperatures increase the probability of crossing climatic tipping points in the earth system dynamics, i.e., positive feedback loops will become more likely (Schellnhuber et al. 2016, Steffen et al. 2016, 2018). This would result in a situation, where returning to the current temperature regime would become unrealistic for future generations.

12. Oceans are currently absorbing around 90 percent of the additional heat (IPCC 2013). They have furthermore absorbed about 30 percent of the $\mathrm{CO}_{2}$ emitted so far. Consequences are rising sea levels, melting of sea ice, acidification and dissolved-oxygen depletion in the oceans. Meeting the goals set by the Paris Agreement is essential to protect humanity and nature, and to mitigate the loss of marine biodiversity and ecosystems, specifically the currently endangered coral communities (IPCC 2018).

13. The human basis of life is threatened in several areas by the crossing of "planetary boundaries". As of 2015, two boundaries are exceeded with a degree of uncertainty (climate and land use change) and two further are critically exceeded: the destruction of genetic variability (biodiversity) and the phosphorus and nitrogen biogeochemical cycles (Steffen et al. 2015).

14. We presently face the largest mass-extinction event since the era of the dinosaurs (Barnosky et al. 2011). Global extinction rates are 100 to 1000 times faster as compared to before humanity exerted its influence (Ceballos et al. 2015, Pimm et al. 2014). The past 500 years saw the extinction of more than 300 land-dwelling vertebrate species (Dirzo et al. 2014); the abundance of investigated vertebrate species has dropped on average by around 60 percent from 1970 to 2014 (WWF 2018).

15. Causes for biodiversity loss are on the one hand habitat destruction by agriculture, deforestation, as well as land consumption by settlements and roads. On the other hand, invasive species play a role, as well as depletion due to over-collection, overfishing and overhunting (Hoffmann et al. 2010).

16. Global warming adds to this: with undiminished $\mathrm{CO}_{2}$ emissions, half of the plant and animal species of the Amazon basin or the Galapagos islands, for example, can be expected to have vanished by 2100 (Warren et al. 2018). Similarly, global warming is the major threat for the survival of coral reefs (Hughes et al. 2017, 2018, IPCC 2018).

17. The loss of agricultural areas and soil fertility, as well as the irreversible destruction of biodiversity and ecosystems threaten the basis of life and limit the options of current and future generations (IPBES 2018a, 2018b, Secretariat of the CBD 2014, Willett et al. 2019, IAAST 2009a, 2009b).

18. Insufficient protection of soil, ocean, fresh-water resources and biodiversity acts as a risk multiplier in the face of global warming (Johnstone and Mazo 2011). It increases the risk that water shortage and famine in many countries will trigger or aggravate social and military conflicts, and contribute to the migration of larger human populations (Levy et al. 2017, World Bank Group 2018, Solow 2013).

19. A sustainable diet with reduced meat, fish and milk consumption, as well as a reorientation of agricultural methods to resource-saving food production are necessary for the protection of land and marine ecosystems and the stabilisation of climate change (Springmann et al. 2018).

20. Meat production produces less than one fifth of the calories used worldwide on more than four fifths of the agricultural area (Poore and Nemecek 2018), and emits a significant proportion of greenhouse gases (FAO 2013). Since the agricultural area includes permanent pastures and meadows as well as croplands, and most of the former cannot be converted to cropland, another comparison is also illustrative: more than one third of the global cereal harvest is used currently as animal feed (FAO 2017).

21. A transition to increased direct consumption of plant-based foods will reduce both the need for cropland and the level of greenhouse gas emissions while providing additional health benefits (Springmann et al. 2016).

22. Direct government subsidies for fossil-based industries amount to more than 100 billion U.S. dollar per year (Jakob et al. 2015). Taking social and environmental costs (in particular health costs, but also air and water pollution) into account, global post-tax subsidies for fossil fuels are significantly higher. According to experts of the International Monetary Fund (IMF) they amount to about five trillion U.S. dollar per year - that is 6.5 percent of global gross domestic product (2014) (Coady et al. 2017).

23. According to the polluter pays principle, the cost of climate damages should be attributed to the burning of fossil fuels. One possible approach is the introduction of $\mathrm{CO}_{2}$ prices. As long as a sufficient supply of low-cost renewable energies is not achieved, the resulting financial burden will need to be distributed in a socially responsible way. Examples are direct transfers or tax reductions for particularly affected households or lump-sum payments for citizens (Klenert et al. 2018).

24. Based on already established sustainable energy technologies, a strong reduction in costs and an increase in production capacities is possible. This would, in turn, render a change from burning fossils to an energy system fully based on renewable energy financially feasible and create new economic possibilities (Nykvist and Nilsson 2015, Creutzig et al. 2017, Jacobson et al. 2018, Teske et al. 2018, Breyer et al. 2018, Löffler et al. 2017 , Pursiheimo et al. 2019).
The young people rightly demand that our society should prioritize sustainability and especially climate action without further hesitation. Without far-reaching and consistent change, their future is in danger. This change means, among other things: we will introduce renewable energy sources with new courage and the necessary speed; we will consistently implement energy-saving measures; and, we will fundamentally change our patterns of nutrition, mobility and consumption. 
Politicians in particular have a responsibility to create the necessary framework conditions in a timely manner. In particular, climate-friendly and sustainable action must become simple and cost-effective, while climate-damaging action must become unattractive and expensive, for example, through effective $\mathrm{CO}_{2}$ pricing (e.g., EFI 2019), elimination of subsidies for climate-damaging actions and products, efficiency regulations and social innovations. A socially balanced distribution of the costs and benefits of change is essential.

The enormous mobilisation of the Fridays for Future/Climate Strike movement shows that young people have understood the situation. As scientists and scholars, we strongly support their demand for rapid and forceful action. As people who are familiar with scientific work and deeply concerned about the current developments, we consider it as our social responsibility to point out the consequences of inadequate action (see also Ripple et al. 2017). Only if we act quickly and consistently can we limit global warming, halt the mass extinction of animal and plant species, preserve the natural basis for life and create a future worth living for present and future generations. This is exactly what the young people of Fridays for Future/Climate Strike are calling for. They deserve our respect and full support. ${ }^{1}$

\section{Die Anliegen der demonstrierenden jungen Menschen sind berechtigt}

Stellungnahme von Wissenschaftlerinnen und Wissenschaftlern aus Deutschland, Österreich und der Schweiz zu den Protesten für mehr Klimaschutz

Z urzeit demonstrieren regelmäßig viele junge Menschen für Klimaschutz und den Erhalt unserer natürlichen Lebensgrundlagen. Als Wissenschaftlerinnen und Wissenschaftler erklären wir auf Grundlage gesicherter wissenschaftlicher Erkenntnisse: Diese Anliegen sind berechtigt und gut begründet. Die derzeitigen Maßnahmen zum Klima-, Arten-, Wald-, Meeres- und Bodenschutz reichen bei weitem nicht aus.

Das Übereinkommen von Paris, meist als Pariser Klimaschutzabkommen bezeichnet, von 2015 (UNFCCC 2015) verpflichtet die Staaten völkerrechtlich verbindlich, die globale Erwärmung deutlich unter $2^{\circ} \mathrm{C}$ zu halten. Darüber hinaus haben alle Länder Anstrengungen versprochen, die Erwärmung auf $1,5^{\circ} \mathrm{C}$ zu begrenzen. Es kommt nun darauf an, die Netto-Emissionen von $\mathrm{CO}_{2}$ und anderen Treibhausgasen schnell abzusenken und weltweit spätestens zwischen 2040 und 2050 auf null zu reduzieren (IPCC 2018). Eine schnellere Absenkung erhöht hierbei die Wahrscheinlichkeit, $1,5^{\circ} \mathrm{C}$ nicht zu überschreiten. Die Verbrennung von Kohle sollte bereits 2030 fast vollständig beendet sein, die Verbrennung von Erdöl und Erdgas gleichzeitig reduziert werden, bis alle

1 The complete lists of initial and final signatories are published as a supplement to this article: www.oekom.de/supplementary-files.html\#c 14564. fossilen Energieträger durch klimaneutrale Energiequellen ersetzt worden sind. Unter Berücksichtigung von globaler Klimagerechtigkeit müsste in Europa dieser Wandel sogar noch deutlich schneller ablaufen (IPCC 2018, Global Carbon Project 2018).

Auch wenn weiterhin Beteiligungs- und Diskussionsbedarf besteht: Jetzt muss gehandelt werden. Beides schließt einander nicht aus. Es gibt bereits viele gesellschaftliche und technologische Innovationen, die Lebensqualität erhalten und menschliches Wohlergehen verbessern können, ohne unsere natürlichen Lebensgrundlagen zu zerstören (siehe zum Beispiel Klima-Allianz Deutschland 2018, WBGU 2011).

In allen deutschsprachigen Ländern werden beim Umbau der Bereiche Energie, Ernährung, Landwirtschaft, Ressourcennutzung und Mobilität die notwendige Größenordnung und Geschwindigkeit nicht erreicht. Deutschland wird die selbstgesteckten Klimaschutzziele für 2020 verfehlen (UBA 2019) und auch die Erreichung der Ziele der Deutschen Nachhaltigkeitsstrategie für 2030 ist hochgradig gefährdet (German Council for Sustainable Development 2018, SRU 2018). Zudem mangelt es weiterhin an einem wirksamen Klimaschutzgesetz. Österreich hat sich in seiner Klima- und Energiestrategie Ziele gesetzt, die dem Pariser Vertrag in keiner Weise gerecht werden (CCCA 2018, Wegener Center für Klima und Globalen Wandel 2018, Schleicher und Kirchengast 2019), und selbst dafür sind weder die erforderlichen Maßnahmen noch die finanziellen Mittel vorgesehen (CCCA 2018). Zugleich sind Bodenverbrauch und -versiegelung pro Person und Jahr in Österreich die höchsten in Europa (UBA 2018). Die Schweiz hat ihre Treibhausgas-Emissionen seit 1990 nur geringfügig verringert; gleichzeitig stiegen die im Ausland verursachten Emissionen erheblich an (BAFU 2018). In der ersten parlamentarischen Debatte zur Totalrevision des $\mathrm{CO}_{2}$-Gesetzes wurden die inländischen Reduktionsziele gestrichen und die Reduzierung der Schweizer Emissionen sollte durch Kompensation im Ausland erfolgen. Schließlich ist das Gesetz vorläufig gescheitert (Schweizer Parlament 2018).

Die jungen Menschen fordern zu Recht, dass sich unsere Gesellschaft ohne weiteres Zögern auf Nachhaltigkeit ausrichtet. Ohne tiefgreifenden und konsequenten Wandel ist ihre Zukunft in Gefahr. Dieser Wandel bedeutet unter anderem: Wir führen mit neuem Mut und mit der notwendigen Geschwindigkeit erneuerbare Energiequellen ein. Wir setzen Energiesparmaßnahmen konsequent um. Und wir verändern unsere Ernährungs-, Mobilitäts- und Konsummuster grundlegend.

Vor allem die Politik steht in der Verantwortung, zeitnah die notwendigen Rahmenbedingungen zu schaffen. Insbesondere muss klimafreundliches und nachhaltiges Handeln einfach und kostengünstig werden, klimaschädigendes Handeln hingegen unattraktiv und teuer, zum Beispiel durch wirksame $\mathrm{CO}_{2}$-Preise (EFI 2019), Einstellung von Subventionen für klimaschädliche Handlungen und Produkte, Effizienzvorschriften und soziale Innovationen. Eine sozial ausgewogene Verteilung von Kosten und Nutzen des Wandels ist dabei unerlässlich.

Die enorme Mobilisierung der neuen Bewegungen (Fridays for Future in Deutschland und Österreich, Klimastreik in der Schweiz) 


\section{BOX: Einige wichtige Fakten}

1. Weltweit ist die Durchschnittstemperatur bereits um etwa $7^{\circ} \mathrm{C}$ angestiegen (relativ zu 1850 bis 1900) (IPCC 2013, 2018). Rund die Hälfte des Anstiegs erfolgte in den letzten 30 Jahren (NASA 2018, IPCC 2014).

2. Weltweit waren die Jahre 2015, 2016, 2017 und 2018 die heißesten Jahre seit Beginn der Wetteraufzeichnungen (NASA 2019).

3. Der Temperaturanstieg ist nahezu vollständig auf die von Menschen verursachten Treibhausgasemissionen zurückzuführen (U.S. Global Change Research Program 2017, IPCC 2013, 2014).

4. Bereits mit der aktuellen Erwärmung sind wir in vielen Regionen mit häufigeren und stärkeren Extremwetterereignissen und deren Folgen wie Hitzewellen, Dürren, Waldbränden und Starkniederschlägen konfrontiert (zum Beispiel IPCC 2012, 2013, 2018, National Academies of Sciences, Engineering, and Medicine 2016)

5. Die Auswirkungen der globalen Erwärmung sind zudem eine Gefahr für die menschliche Gesundheit (Watts et al. 2015, 2018). Neben den oben genannten direkten Folgen sind dabei auch indirekte Folgen der globalen Erwärmung wie Ernährungsunsicherheit und die Verbreitung von Krankheitserregern und -überträgern zu beachten.

6. Falls die Weltgemeinschaft die vom Pariser Klimaschutzabkommen angestrebte Beschränkung der Erwärmung auf $1,5^{\circ} \mathrm{C}$ verfehlt, ist in vielen Regionen der Welt mit erheblich verstärkten Klimafolgen für Mensch und Natur zu rechnen (IPCC 2018).

7. Um mit hoher Wahrscheinlichkeit eine Erwärmung von $1,5^{\circ} \mathrm{C}$ nicht zu überschreiten, müssen die Nettoemissionen von Treibhausgasen (insbesondere $\mathrm{CO}_{2}$ ) sehr rasch sinken und in den nächsten 20 bis 30 Jahren weltweit auf null reduziert werden (IPCC 2013, 2018).

8. Stattdessen steigen die $\mathrm{CO}_{2}$-Emissionen weiter. Mit den Vorschlägen, die weltweit derzeit auf dem Tisch liegen, wird die Erwärmung bis zum Ende des Jahrhunderts wahrscheinlich bei über $3^{\circ} \mathrm{C}$ liegen und anschließend aufgrund anhaltender Emissionen und Rückkopplungseffekte weiter zunehmen (Climate Action Tracker 2018).

9. Bei derzeitigen Emissionen reicht das verbleibende globale $\mathrm{CO}_{2}$-Emissionsbudget für den 1,5-Grad-Pfad nur für etwa zehn Jahre. Auch für den 2-Grad-Pfad reicht es nur für etwa 25 bis 30 Jahre (MCC 2018, IPCC 2018).

10. Anschließend leben wir von einem „ $\mathrm{CO}_{2}$-Überziehungskredit", das heißt, die ab dann emittierten Treibhausgase müssen später unter großen Anstrengungen wieder aus der Atmosphäre entfernt werden (zum Beispiel Rogelj et al. 2018, Gasser et al. 2015). Bereits die heute lebenden jungen Menschen sollen diesen „Kredit“ wieder abbezahlen. Gelingt dies nicht, werden viele nachfolgende Generationen unter den gravierenden Folgen der Erderwärmung leiden.

11. Bei zunehmender Erwärmung der Erde werden gefährliche klimatische Kipp-Punkte des Erdsystems, also sich selbst verstärkende Prozesse, immer wahrscheinlicher (Schellnhuber et al. 2016, Steffen et al. 2016, 2018). Dies würde dazu führen, dass eine Rückkehr zu heutigen globalen Temperaturen für kommende Generationen nicht mehr realistisch ist.

12. Die Ozeane nehmen zurzeit rund 90 Prozent der zusätzlichen Wärme auf (IPCC 2013). Sie haben zudem etwa 30 Prozent des bisher emittierten $\mathrm{CO}_{2}$ aufgenommen. Die Konsequenzen sind Meeresspiegelanstieg, Verlust von Meereis, Versauerung und Sauerstoffmangel im Ozean. Die konsequente Umsetzung der Ziele des Pariser Klimaschutzabkommens ist essenziell, um Mensch und Natur zu schützen und den Verlust von marinen Arten und Lebensräumen, besonders der akut gefährdeten Korallenriffe, zu begrenzen (IPCC 2018).

13. In vielen Bereichen werden menschliche Lebensgrundlagen durch Überschreitung der planetaren Belastungsgrenzen gefährdet (Steffen et al. 2015, SRU 2016). Mit Stand 2015 sind zwei der neun Grenzen bedenklich überschritten (Klimaerwärmung, Landnutzungsänderungen), zwei weitere (Zerstörung genetischer Vielfalt [Biodiversität], Belastung der Phosphorund Stickstoffkreisläufe) kritisch überschritten (Steffen et al. 2015)

14. Zurzeit findet das größte Massenaussterben seit dem Zeitalter der Dinosaurier statt (Barnosky et al. 2011). Weltweit sterben Arten derzeit hundertbis tausendmal schneller aus als vor dem Beginn menschlicher Einflüsse (Ceballos et al. 2015, Pimm et al. 2014). In den letzten 500 Jahren sind über
300 Landwirbeltierarten ausgestorben (Dirzo et al. 2014); die untersuchten Bestände von Wirbeltierarten sind zwischen 1970 und 2014 im Durchschnitt um 60 Prozent zurückgegangen (WWF 2018).

15. Gründe für den Rückgang der Biodiversität sind zum einen Lebensraumverluste durch Landwirtschaft, Entwaldung und Flächenverbrauch für Siedlung und Verkehr. Zum anderen sind es invasive Arten sowie Übernutzung in Form von Übersammlung, Überfischung und „Überjagung“ (Hoffmann et al. 2010).

16. Die Erderwärmung kommt hinzu: Bei unveränderten $\mathrm{CO}_{2}$-Emissionen könnten bis 2100 zum Beispiel aus dem Amazonasbecken oder von den Galapagosinseln die Hälfte der Tier- und Pflanzenarten verschwinden (Warren et al. 2018). Auch für die tropischen Korallenriffe ist die Meereserwärmung der Hauptbedrohungsfaktor (Hughes et al. 2017, 2018, IPCC 2018).

17. Auch der Verlust an landwirtschaftlicher Nutzfläche und Bodenfruchtbarkeit sowie die irreversible Zerstörung von Artenvielfalt und Ökosystemen gefährden die Lebensgrundlagen und Handlungsoptionen heutiger und kommender Generationen (IPBES 2018a, 2018b, Secretariat of the CBD 2014, Willett et al. 2019, IAAST 2009a, 2009b).

18. Insgesamt besteht durch unzureichenden Schutz der Böden, Ozeane, Süßwasserressourcen und Artenvielfalt - bei gleichzeitiger Erderwärmung als „Risikovervielfacher" (Johnstone und Mazo 2011) - die Gefahr, dass Trinkwasser- und Nahrungsmittelknappheit in vielen Ländern soziale und militärische Konflikte auslösen oder verschärfen und zur Migration größerer Bevölkerungsgruppen beitragen (Levy et al. 2017, World Bank Group 2018 , Solow 2013).

19. Eine nachhaltige Ernährung mit starker Reduzierung unseres Fisch-, Fleischund Milchkonsums und eine Neuausrichtung der Landwirtschaft auf ressourcenschonende Lebensmittelproduktion sind für den Schutz des Klimas, der Land- und Meeresökosysteme notwendig (Springmann et al. 2018).

20. Nutztierhaltung erzeugt auf über vier Fünftel der landwirtschaftlich genutzten Fläche weniger als ein Fünftel der weltweit konsumierten Kalorien (Poore und Nemecek 2018) und hat einen erheblichen Anteil am Ausstoß klimaschädlicher Treibhausgase (FAO 2013). Da die landwirtschaftlich genutzte Fläche Dauergrünland, Dauerkulturen und Ackerflächen umfasst und ein erheblicher Teil des Dauergrünlands nicht in Ackerland verwandelt werden kann, ist auch folgender Vergleich relevant: Über ein Drittel der weltweiten Getreideernte wird zurzeit als Tierfutter verwendet (FAO 2017).

21. Ein verstärkter Direktkonsum von pflanzlicher Nahrung reduziert den Bedarf an knapper Ackerfläche, erzeugt weniger Treibhausgase und hat zudem erhebliche gesundheitliche Vorteile (Springmann et al. 2016).

22. Die direkten staatlichen Subventionen für fossile Brennstoffe betragen jährlich mehrere 100 Milliarden US-Dollar (Jakob et al. 2015). Berücksichtigt man zusätzlich noch die nicht durch Steuern ausgeglichenen Sozial- und Umweltkosten (vor allem Gesundheitskosten durch Luftverschmutzung), wird die Nutzung fossiler Brennstoffe nach Schätzungen von Experten des Internationalen Währungsfonds (IMF) weltweit mit rund fünf Billionen US-Dollar pro Jahr unterstützt; das sind 6,5 Prozent des weltweiten Bruttoinlandsprodukts von 2014 (Coady et al. 2017).

23. Um dem Verursacherprinzip Rechnung zu tragen, müssten die Klimaschäden den Kosten der Verbrennung fossiler Brennstoffe zugerechnet werden. Eine Methode, mit der die Emissionen besonders effizient gesenkt werden können, sind zum Beispiel $\mathrm{CO}_{2}$-Preise. Solange eine Versorgung durch kostengünstige erneuerbare Energieformen noch nicht ausreichend erreicht ist, müssen die dadurch entstehenden Belastungen sozialverträglich gestaltet werden. Dies ist etwa durch Transferzahlungen oder Steuererleichterungen für besonders betroffene Haushalte oder eine pauschale Auszahlung an die Bürgerinnen und Bürger möglich (Klenert et al. 2018).

24. Stark sinkende Kosten und steigende Produktionskapazitäten für bereits eingeführte klimafreundliche Technologien machen eine Abkehr von fossilen Brennstoffen hin zu einem vollständig auf erneuerbaren Energien basierenden Energiesystem bezahlbar und schaffen neue ökonomische Chancen (Nykvist and Nilsson 2015, Creutzig et al. 2017, Jacobson et al. 2018, Teske et al. 2018, Breyer et al. 2018, Löffler et al. 2017, Pursiheimo et al. 2019). 
zeigt, dass die jungen Menschen die Situation verstanden haben. Ihre Forderung nach schnellem und konsequentem Handeln können wir als Wissenschaftlerinnen und Wissenschaftler nur nachdrücklich unterstreichen.

Als Menschen, die mit wissenschaftlichem Arbeiten vertraut sind und denen die derzeitigen Entwicklungen große Sorgen bereiten, sehen wir es als unsere gesellschaftliche Verantwortung an, auf die Folgen unzureichenden Handelns hinzuweisen (siehe auch Ripple et al. 2017). Nur wenn wir rasch und konsequent handeln, können wir die Erderwärmung begrenzen, das Massenaussterben von Tier- und Pflanzenarten aufhalten, die natürlichen Lebensgrundlagen bewahren und eine lebenswerte Zukunft für derzeit lebende und kommende Generationen gewinnen. Genau das möchten die jungen Menschen von Fridays for Future und Klimastreik erreichen. Ihnen gebührt unsere Achtung und unsere volle Unterstützung.

\section{Process and Results ${ }^{2}$}

Since 2018, several youth movements, such as Fridays for Future in Germany and Austria or Climate Strike in Switzerland call for immediate and decisive climate and sustainability action. They are adamant that their demands are firmly based on the results of scientific studies. In the spring of 2019, several participants in this movement were being defamed. Faced with children and young adults who began to politically fight for their right for a sustainable, peaceful future, many media outlets and politicians did not engage with the substance of the demands. Rather, they preferred to question the forms of protests and the competence of the young people (von Lucke 2019).

Following the lead of a Belgian initiative (Vicca et al. 2019), a small group of German speaking scientists and scholars decided to pro-actively analyse the assumptions and demands of the young protesters, and to counter false and conspiracy-theory-based interventions. The question, whether this action might strengthen or weaken the youth movement, was initially controversially debated within the team. However, the plans were discussed with members of the movement and they welcomed them. A time plan was developed aimed at not unduly diluting media attention away from the youth movement.

The statement and the associated selection of facts was prepared within four weeks by scientists from Germany, Austria, and Switzerland, with a broad diversity of backgrounds and at various stages of their careers. It was then circulated by email for two weeks and released at several press conferences on March 12, 2019 (with members of the youth movement as guests). The open signing period ended ten days after the press conference, at which point it had gathered over 26,800 signatures from scientists. ${ }^{3}$

All who signed did this on their personal behalf and not on that of their affiliated institutions. Every signee was required to indicate the level of present or past direct involvement in science or scholarship, particularly, whether having scientifically published or not. We verified email addresses, checked the data for systematic errors and falsifications, and scrutinized a sample of almost 500 signees in detail. ${ }^{4}$ A large fraction of the signees had published scientific or scholarly works (71.1 percent) and a further 24.8 percent are currently actively working in science and academia (e.g., PhD candidates). Of all those who signed, four percent belong to the categories Degree at the level of a Master without publications or Citizen Scientists without publications. 63 percent of all signees have a doctorate or professorship.

As intended, signees come from a broad diversity of scientific and scholarly disciplines. We consider it necessary to form an alliance that goes far beyond specialists in climate and biodiversity science, sustainability, social science, or engineering. We will not achieve a sustainable future without, for example, including aspects of political participation, education, gender, and justice issues (including climate justice). We need the diverse gifts, experiences, and insights of all disciplines to solve the unprecedented problems that humanity is facing.

The statement is not a petition to government and politics. Like all scientific or scholarly publications, it addresses the public. In open, democratic societies, all citizens are entitled to sufficient knowledge so that they have the opportunity to participate competently in the discussion of public affairs and guide and control the professionalized exercise of power.

Politics has acknowledged the contribution of Scientists for Future to the political debate. On March 15, 2019 the German Bundestag held a session on The Federal Government's attitude to the climate strikes of the Fridays for Future movement and the Scientists for Future petition (Deutscher Bundestag 2019). In the weeks afterwards, members from Scientists for Future were invited for talks by several parties on the federal and local level. In Austria, the initiators of the Fridays for Future movement entered into discussions with the Federal President, the Federal Minister for Sustainability and Tourism and the Federal Minister for Education, Science and Research. They called on the Federal President to convene a committee of political decision-makers and scientists. ${ }^{5}$ In Switzerland, several cantons have declared a climate emergency and the Swiss Freisinnig-demokratische Partei (FDP) has announced a turnaround in climate policy (Neuhaus 2019).

Around the same time, several independently organized statements or letters in support of the youth movement were published in other countries. The organizers of these statements or letters then formed an alliance to also publish a joint, international statement (Hagedorn et al. 2019) on April 12, 2019.

\footnotetext{
2 The following text was not part of the original statement signed by over 26,800 scientists and scholars.

3 The distribution was 21,679 from Germany, 2,773 from Switzerland, and 2,222 from Austria, plus 129 from other German-speaking regions.

4 About three percent of all signatures were rejected. Only a very small fraction involved wilful falsifications. The majority of rejections were situations where the provided information was insufficient to judge whether the signees were indeed scientists or scholars.

5 www.bundespraesident.at/aktuelles/detail/news/fridays
} 
As an unfunded, small, grassroots, volunteer group without institutional support, Scientists for Future had very limited means. The fact that it gained so much recognition in such a short time span and that so many people volunteered in spreading the word to our colleagues indicates that their statement resonates strongly with many scientists and scholars.

\section{The statement and the demands of Fridays for Future}

On April 8, 2019, the German Fridays for Future movement released a catalogue of demands for climate action (Fridays for Future 2019). This catalogue was prepared over several months by a working group of the youth movement. Scientists for Future had provided reviews of draft versions of the demand catalogue. Differences between the demands and our statement exist. For example, to limit global warming to $1.5^{\circ} \mathrm{C}$ above pre-industrial levels, we conclude in accordance with IPCC (2018) that net zero emissions will have to be reached globally between 2040 and 2050 at the latest, whereas Fridays for Future demands net zero to be reached in Germany by 2035.

This is not a contradiction, since we consider it scholarly justified to include aspects of climate justice, that is, that different countries face different challenges and responsibilities. Strong, industrialized countries have more capabilities to be leaders and innovators in the transformation process. At the same time, these countries have a higher responsibility based on their historic emissions. Thus, industrialized countries should not only bear a greater contribution of the costs (Kartha et al. 2018), they also need to act faster to allow poorer and less developed countries to follow without undue risks to their economy and development. In our statement, we discussed the global carbon budgets of IPCC (2018) and its implications. Fridays for Future derived a demand for Germany which includes aspects of climate justice.

\section{Reflection}

In the increasingly complex and interwoven relation between humans and the earth system, scientists and scholars play a critical role in knowledge production and application and are called upon to actively feed their knowledge into the public arenas of opinionforming (Jahn 2013). The relations between the expert knowledge sphere, the public sphere, and the sphere of political decision-making are complex - for good reasons (Heidenreich 2018).

When reflecting on our own understanding of the relationship between experts and the political processes, we assert that the mode of interaction must depend 1. on the extent of risks that humans are exposed to as a consequence of a decision, and 2. on the time available for corrective action. To consider an example: experts consulted about a transportation issue may conclude that it would be best to build a bridge of a specified quality at a certain place. The political process may come to a wide variety of conclu- sions: build no bridge at all, build it elsewhere, or build a cheaper bridge with higher maintenance costs and a shorter lifespan. Such decisions justify a critical expert publication, but little more. However, when it is decided to build a bridge that is liable to break in unpredictable ways or emits poisonous substances endangering the livelihood of local communities, a different role for scientists and scholars is called for. This difference is not about questioning the precedence of the democratic process, but about fulfilling an obligation to society through pro-active dissemination of knowledge. Just like medical experts have an ethical duty to warn of an impending epidemic, we consider it our ethical obligation to raise our voices to warn about the dangers of climate change, pollution and biosphere degradation.

The findings of earth system sciences over the past decades have clearly shown that climate change, degradation of the Earth's biosphere, and environmental pollution are caused by human societies and are approaching or, in some cases, have already transgressed thresholds that many consider dangerous or associated with high risks (e.g., IPCC 2014, Ceballos et al. 2015). These conclusions have led to many international agreements on biodiversity, climate, and sustainability, such as the Aichi Targets to halt and reverse biodiversity loss, the Paris Agreement of the UN Framework Convention on Climate Change, and the UN 2030 Agenda, respectively the Sustainable Development Goals (SDGs). Unfortunately, many scientific, social, and economic parameters indicate that these agreements are currently not sufficiently translated into political action and economic and societal practice. In order to counteract the risks of crossing thresholds and irreversible tipping points in the Earth system (Steffen et al. 2015), societies urgently need to transform fundamentally towards sustainable practices.

With respect to the topic of time, we understand that it is important to take the time to understand consequences of political decisions (Heidenreich 2018). However, for the preservation of the natural foundations of life, acting without sufficient speed has serious consequences. Humanity cannot simply press a "pause button" in the ongoing accumulation of greenhouse gases in the atmosphere, the degradation of ecosystems and soils, or the drastic reduction of species populations leading to extinctions.

For example, a frequently encountered assumption is that the magnitude of climate change would depend primarily on the volume of emissions produced today. In reality, however, it depends on the overall history of these emissions, that is, on the total accumulated emissions over time. Consequently, postponement of action will not just delay a solution but effectively result in stronger adverse climate forcing.

Humanity is aware of the problems of global warming for more than half a century. Revelle et al. (1965) warned in an official US government report of rising sea levels due to $\mathrm{CO}_{2}$ emissions and recommended "economic incentives to discourage pollution" in which "special taxes would be levied against polluters" (Revelle et al. 1965). But while humanity is divided about the available options and best ways forward, it is, in practice, taking what we consider the dangerous decision to continue business as usual. We carry on limiting our response to debates and largely symbolic actions 
(or, in the case of scholars, communicating the need for societal change mostly in scholarly journals and communities). Similarly to how non-communication is communication (Watzlawick et al. 1967), a non-decision is a decision.

We perceive our statement here as a scientifically substantiated warning about the probable consequences of this concrete decision, not as a statement of impatience with political processes in general. We fully endorse our political constitution. We do, however, believe that our democratic system can and needs to undertake evolutionary changes to its collective time management (Heidenreich 2018), both in terms of agility and in terms of re-adjusting the balance between short-term and long-term benefits and costs. Scientists for Future is an unfunded volunteer expert group, not a political campaigning group. As scientists and scholars, we are committed to distinguish between political conviction and scientific and scholarly results. We are aware that this is not easy because research is not immune to political influence. Researchers may work for publicly funded academic institutions, governments, corporations, companies or NGOs. They work in frameworks deciding where to invest resources, which money to accept, and which fraction of research to highlight in their science communication. Furthermore, everyone has personal ethical and political convictions. The conclusion should not be to separate research from society, but to embrace a framework of responsible research that included societal and ethical reflections (Helming et al. 2016).

Our professional ethos does not limit us to speak only when asked. We believe that while our societies must become more scientific, our scientists must become more socially (and politically) aware (Jahn et al. 2015). The motivation to review the assumptions of the youth movement is based on ethical considerations and, in consequence, political. We do, however, hold ourselves accountable to the scientific process of review and transparency. Accordingly, our statement is not the result of prior opinions, but of a painstaking review process that ensured that the statement is scientifically well founded. Producing and disseminating knowledge is part of shaping societies. Whether we speak or remain silent: we are part of the political debate. Remaining neutral and silent about our established state of knowledge on global environmental change would be a violation of our professional responsibilities towards our societies.

We thank Adam Wilkins, Rob Stevenson, Adina Arth, Jens Jetzkowitz and Johannes Fischer for their support in improving this text.

\section{References}

BAFU (Bundesamt für Umwelt). 2018. Klimapolitik der Schweiz: Umsetzung des Abkommens von Paris. www.bafu.admin.ch/bafu/de/home/themen/ klima/publikationen-studien/publikationen/klimapolitik-der-schweiz.html (accessed March 11, 2019).

Barnosky, A. D. et al. 2011. Has the Earth's sixth mass extinction already arrived? Nature 471: 51-57.

Breyer, C. et al. 2018. Solar photovoltaics demand for the global energy transition in the power sector. Progress in Photovoltaics: Research and Applications 26/8: 505-523.
CCCA (Climate Change Centre Austria). 2018. Stellungnahme zum Konsultationsentwurf des Nationalen Energie- und Klimaplans (NEKP). https://wegcwww.uni-graz.at/publ/downloads/CCCA_NKK-Wiss_ Stellungnahme-NEKP_6Dez2018.pdf (accessed April 12, 2019).

Ceballos, G., P. R. Ehrlich, A. D. Barnosky, A. García, R. M. Pringle, T. M. Palmer. 2015. Accelerated modern human-induced species losses: Entering the sixth mass extinction. Science Advances 1/5: e1400253-e1400253.

Climate Action Tracker. 2018. Temperatures: Addressing global warming. https://climateactiontracker.org/global/temperatures (accessed April 12, 2019).

Coady, D., I. Parry, L. Sears, B. Shang. 2017. How large are global fossil fuel subsidies? World Development 91/C: 11-27.

Creutzig, F., P. Agoston, J. C. Goldschmidt, G. Luderer, G. Nemet, R. C. Pietzcker. 2017. The underestimated potential of solar energy to mitigate climate change. Nature Energy 2: 17140.

Deutscher Bundestag. 2019. Beeindruckt von Klimastreiks der „Fridays for Future“-Bewegung. www.bundestag.de/dokumente/textarchiv/2019/ kw11-de-aktuelle-stunde-klimastreiks-628294 (accessed April 29, 2019).

Dirzo, R., H. S. Young, M. Galetti, G. Ceballos, N. J. B. Isaac, B. Collen. 2014. Defaunation in the Anthropocene. Science 345/6195: 401-406.

EFI (Expertenkommission Forschung und Innovation). 2019. Gutachten 2019. www.e-fi.de/fileadmin/Gutachten_2019/EFI_Gutachten_2019.pdf (accessed, April 12, 2019).

FAO (Food and Agriculture Organization of the United Nations). 2013. Tackling climate change through livestock: A global assessment of emissions and mitigation opportunities. www.fao.org/3/a-i3437e.pdf (accessed April 12, 2019).

FAO. 2017. Food balance sheets. Data for 2013. www.fao.org/faostat/en/\#data/FBS (accessed April 3, 2019).

Fridays for Future. 2019. Unsere Forderungen an die Politik. https://fridaysforfuture.de/forderungen (accessed May 9, 2019).

Gasser, T., C. Guivarch, K. Tachiiri, C. D. Jones, P. Ciais. 2015. Negative emissions physically needed to keep global warming below $2^{\circ} \mathrm{C}$. Nature Communications 6: 7958.

German Council for Sustainable Development. 2018. The 2018 peer review on the German Sustainability Strategy. www.nachhaltigkeitsrat.de/ wp-content/uploads/2018/05/2018_Peer_Review_of_German_ Sustainability_Strategy_BITV.pdf (accessed April 10, 2019).

Global Carbon Project. 2018. Global carbon budget 2018. www.globalcarbonproject.org/carbonbudget/18/files/ GCP_CarbonBudget_2018.pdf (accessed February 27, 2019).

Hagedorn, G. et al. 2019. Concerns of young protesters are justified. Science 364: 139-140. DOI: 10.1126/science.aax3807.

Heidenreich, F. 2018. How will sustainability transform democracy? Reflections on an important dimension of transformation sciences. GAIA 27/4: 357-362. DOI: 10.14512/gaia.27.4.7.

Helming, K. et al. 2016. Forschen für nachhaltige Entwicklung. Kriterien für gesellschaftlich verantwortliche Forschungsprozesse. GAIA 25/3: 161-165. DOI: 10.14512/gaia.25.3.6.

Hoffmann, M. et al. 2010. The impact of conservation on the status of the world's vertebrates. Science 330/6010: 1503-1509.

Hughes, T.P. et al. 2017. Coral reefs in the Anthropocene. Nature 546: 82-90.

Hughes, T.P. et al. 2018. Spatial and temporal patterns of mass bleaching of corals in the Anthropocene. Science 359/6371: 80-83.

IAAST (International Assessment of Agricultural Knowledge, Science and Technology for Development). 2009a. Weltagrarbericht: Synthesebericht. www.weltagrarbericht.de/fileadmin/files/weltagrarbericht/IAASTDBerichte/IAASTDSyntheseDeutsch.pdf (accessed April 12, 2019).

IAAST. 2009b. Agriculture at a crossroads. www.weltagrarbericht.de/ fileadmin/files/weltagrarbericht/IAASTDBerichte/SynthesisReport.pdf. (accessed April 12, 2019).

IPBES (Intergovernmental Science-Policy Platform on Biodiversity and Ecosystem Services). 2018a. The assessment report on land degradation and restoration. www.ipbes.net/system/tdf/2018_Idr_full_report_book_v4_ pages.pdf?file=1\&type=node\&id=29395 (accessed March 10, 2019).

IPBES. 2018b. The regional assessment report on biodiversity and ecosystem services for Africa. www.ipbes.net/system/tdf/africa_assessment_report_ 20181219_0.pdf?file=1\&type=node\&id=29243 (accessed March 10, 2019). 
IPCC (Intergovernmental Panel on Climate Change). 2012. Managing the risks of extreme events and disasters to advance climate change adaptation: Special report. www.ipcc.ch/site/assets/uploads/2018/03/SREX_Full_Report-1.pdf (accessed April 12, 2019).

IPCC. 2013. Climate change 2013: The physical science basis. Contribution of working group I to the fifth assessment report of the Intergovernmental Panel on Climate Change. Cambridge, UK: Cambridge University Press.

IPCC. 2014. Climate change 2014: Synthesis report. Contribution of working groups I, II and III to the fifth assessment report of the Intergovernmental Panel on Climate Change. Geneva: IPCC, www.ipcc.ch/site/assets/ uploads/2018/02/SYR_AR5_FINAL_full.pdf (accessed May 16, 2019).

IPCC. 2018. Global warming of $1.5^{\circ} \mathrm{C}$. Special report. www.ipcc.ch/report/sr 15 (accessed April 12, 2019).

Jacobson, M.Z., M. A. Delucchi, M. A. Cameron, B. V. Mathiesen. 2018. Matching demand with supply at low cost in 139 countries among 20 world regions with 100\% intermittent wind, water, and sunlight (WWS) for all purposes. Renewable Energy 123: 236-248.

Jahn, T. 2013. Wissenschaft für eine nachhaltige Entwicklung braucht eine kritische Orientierung. GAIA 22/1: 29-33. DOI: 10.14512/gaia.22.1.9.

Jahn, T. et al. 2015. Nachhaltige Wissenschaft im Anthropozän. GAIA 24/2: 92-95. DOI: 10.14512/gaia.24.2.6.

Johnstone, S., J. Mazo. 2011. Global warming and the Arab spring. Survival 53/2:11-17.

Jakob, M., C. Chen, S. Fuss, A. Marxen, O. Edenhofer. 2015. Development incentives for fossil fuel subsidy reform. Nature Climate Change 5: 709-712.

Kartha, S., S. Caney, N. K. Dubash, G. Muttitt. 2018. Whose carbon is burnable? Equity considerations in the allocation of a "right to extract". Climatic Change 150: 117-129. DOI: 10.1007/s10584-018-2209-z.

Klenert, D. et al. 2018. Making carbon pricing work for citizens. Nature Climate Change 8: 669-677.

Klima-Allianz Deutschland. 2018. Wann, wenn nicht jetzt: Das Maßnahmenprogramm Klimaschutz 2030 der deutschen Zivilgesellschaft. www.klima-allianz.de/fileadmin/user_upload/Maßnahmenprogramm2030_web.pdf (accessed April 10, 2019).

Levy, B. S., V.W. Sidel, J.A. Patz. 2017. Climate change and collective violence. Annual Review of Public Health 38: 241-257.

Löffler, K., K. Hainsch, T. Burandt, P. Y. Oei, C. Kemfert, C. von Hirschhausen. 2017. Designing a model for the global energy system - GENeSYS-MOD: An application of the Open-Source Energy Modeling System (OSeMOSYS). Energies 10/10: 1-28.

MCC (Mercator Research Institute on Global Commons and Climate Change). 2018. So schnell tickt die $\mathrm{CO}_{2}$-Uhr. www.mcc-berlin.net/de/forschung/ co2-budget.html (accessed April 10, 2019).

NASA (National Aeronautics and Space Administration). 2018. Annual mean land-ocean temperature index in 0.01 degrees Celsius selected zonal means. https://data.giss.nasa.gov/gistemp/tabledata_v3/ZonAnn.Ts+dSST.txt (accessed April 12, 2019).

NASA. 2019. 2018 fourth warmest year in continued warming trend, according to NASA, NOAA. https://climate.nasa.gov/news/2841/ 2018-fourth-warmest-year-in-continued-warming-trend-according-tonasa-noaa (accessed April 12, 2019).

National Academies of Sciences, Engineering, and Medicine (Ed.). 2016 Attribution of extreme weather events in the context of climate change. Washington, D.C.: National Academies Press.

Neuhaus, C., 2019. Die FDP plant in der Klimapolitik den Befreiungsschlag. Neue Züricher Zeitung. www.nzz.ch/schweiz/die-fdp-plant-in-derklimapolitik-den-befreiungsschlag-Id.1460137 (accessed May 16, 2019).

Nykvist, B., M. Nilsson. 2015. Rapidly falling costs of battery packs for electric vehicles. Nature Climate Change 5: 329-332.

Pimm, S. L. et al. 2014. The biodiversity of species and their rates of extinction, distribution, and protection. Science 344/6187.

Poore, J., T. Nemecek. 2018. Reducing food's environmental impacts through producers and consumers. Science 360/6392: 987-992.

Pursiheimo, E., H. Holttinen, T. Koljonen. 2019. Inter-sectoral effects of high renewable energy share in global energy system. Renewable Energy 136: 1119-1129.

Revelle, R. et al. 1965. Restoring the quality of our environment. Report of the Environmental Pollution Panel, President's Science Advisory Committee. Washington, D. C.: The White House. 111-133.
Ripple, W. J. et al. 2017. World scientists' warning to humanity: A second notice. BioScience 67/12: 1026-1028.

Rogelj, J. et al. 2018. Scenarios towards limiting global mean temperature increase below $1.5^{\circ} \mathrm{C}$. Nature Climate Change 8: 325-332.

Schellnhuber, H. J., S. Rahmstorf, R. Winkelmann. 2016. Why the right climate target was agreed in Paris. Nature Climate Change 6: 649-653.

Schleicher, S., G. Kirchengast. 2019. Monitoring der österreichischen Treibhausgasemissionen bezüglich der im Klimaschutzgesetz festgelegten Höchstmengen. https://wegcwww.uni-graz.at/publ/downloads/KSG-MonitoringNowcasting_Memo-WEGC_v3-5Mar2019.pdf (accessed April 12, 2019).

Schweizer Parlament. 2018. Nationalrat lehnt verwässertes $\mathrm{CO}_{2}$-Gesetz $a b$. www.parla_ment.ch/de/services/news/Seiten/2018/2018121111 5905786194158159041_bsd067.aspx (accessed February 28, 2019).

Secretariat of the CBD (Convention on Biological Diversity). 2014. Global biodiversity outlook 4: A mid-term assessment of progress towards the implementation of the strategic plan for biodiversity 2011-2020. http://wedocs.unep.org/bitstream/handle/20.500.11822/9261/ gbo4-en.pdf? sequence=8\&isAllowed=y (accessed April 12, 2019).

Solow, A. R. 2013. Global warming: A call for peace on climate and conflict. Nature 497: 179-180.

Springmann, M., H.C.J. Godfray, M. Rayner, P. Scarborough. 2016. Analysis and valuation of the health and climate change cobenefits of dietary change. Proceedings of the Natural Academy of Sciences of the United States of America 113/15: 4146-4151.

Springmann, M. et al. 2018. Options for keeping the food system within environmental limits. Nature 562: 519-525.

SRU (Sachverständigenrat für Umweltfragen). 2018. Umweltziele der Nachhaltigkeitsstrategie drohen weitgehend verfehlt zu werden. www.umweltrat.de/ SharedDocs/Kurzmeldungen/DE/2016_2020/2018_06_Umweltziele_ Nachhaltigkeitsstrategie.html?nn=9724688 (accessed April 12, 2019).

Steffen, W. et al. 2015. Planetary boundaries: Guiding human development on a changing planet. Science 347/6223: 736-747.

Steffen, W. et al. 2016. Stratigraphic and earth system approaches to defining the anthropocene. Earth's Future 4/8: 324-345.

Steffen, W. et al. 2018. Trajectories of the earth system in the Anthropocene. Proceedings of the National Academy of Sciences of the United States of America 115/33: 8252-8259.

Teske, S., T. Pregger, S. Simon, T. Naegler. 2018. High renewable energy penetration scenarios and their implications for urban energy and transport systems. Current Opinion in Environmental Sustainability 30: 89-102.

UBA (Umweltbundesamt). 2018. Flächeninanspruchnahme. www.umweltbundesamt.at/umweltsituation/raumordnung/ rp_flaecheninanspruchnahme (accessed April 12, 2019).

UBA. 2019. Indikator: Emissionen von Treibhausgasen. www.umweltbundesamt.de/ indikator-emission-von-treibhausgasen\#textpart-1 (accessed February 26, 2019).

UNFCCC (United Nations Framework Convention on Climate Change). 2015. Paris Agreement. https://unfccc.int/files/essential_background/convention/ application/pdf/english_paris_agreement.pdf (accessed April 12, 2019).

U.S. Global Change Research Program. 2017. Climate science special report: Fourth National Climate Assessment. Volume I. https://doi.org/10.7930/J0J964/6 (accessed April 12, 2019).

Vicca, S. et al. 2019. Scientists4Climate. www.scientists4climate.be (accessed April 29, 2019)

von Lucke, A. 2019. "Fridays for Future": Der Kampf um die Empörungshoheit. Wie die junge Generation um ihre Stimme gebracht werden soll. Blätter für deutsche und internationale Politik 64/3: 91-100.

Warren R., J. Price, J. VanDerWal, S. Cornelius, H. Sohl. 2018. The implications of the United Nations Paris Agreement on Climate Change for Globally Significant Biodiversity Areas. Climatic Change 147/3 - 4: 395-409.

Watts, N. et al. 2015. Health and climate change: policy responses to protect public health. Lancet 386/10006: 1861-1914.

Watts, N. et al. 2018. The 2018 report of the Lancet Countdown on health and climate change: Shaping the health of nations for centuries to come. Lancet 392/10163: 2479-2514.

Watzlawick, P., J. B. Bavelas, D. D. Jackson. 1967. Pragmatics of human communication: A study of interactional patterns, pathologies, and paradoxes. New York: Norton. 
WBCU (Wissenschaftlicher Beirat der Bundesregierung Globale Umweltveränderungen). 2011. Welt im Wandel: Gesellschaftsvertrag für eine Große Transformation. Hauptgutachten 2011. Berlin: WBGU.

Wegener Center für Klima und Globalen Wandel. 2018. Stellungnahme zum Entwurf der Klima- und Energiestrategie (KES) für Österreich. https://wegcwww.uni-graz.at/publ/downloads/Stellungnahme_KES_ WegenerCenter.pdf (accessed April 12, 2019).

Willett, W. et al. 2019. Food in the Anthropocene: The EAT-Lancet Commission on healthy diets from sustainable food systems. Lancet 393/10170: 447-492.

World Bank Group. 2018. Groundswell: Preparing for internal climate migration. https://openknowledge.worldbank.org/handle/10986/29461 (accessed March 1, 2019).

WWF (World Wide Fund For Nature). 2018. Living planet report 2018 . Aiming higher. http://wwf.panda.org/knowledge_hub/all_publications/ living_planet_report_2018 (accessed April 12, 2019).

\section{CONTRIBUTING AUTHORS}

Dr. Gregor Hagedorn

Berlin, Germany

Thomas Loew

Institute for Sustainability, Berlin, Germany

Prof. Dr. Sonia I. Seneviratne

ETH Zurich, Zurich, Switzerland

Prof. Dr. Wolfgang Lucht

Potsdam Institute for Climate Impact Research, Potsdam, Germany

Marie-Luise Beck

German Climate Association, Berlin, Germany

Dr. Janina Hesse

Humboldt-Universität zu Berlin, Berlin, Germany

Prof. Dr. Reto Knutti

ETH Zurich, Zurich, Switzerland

Prof. Dr. Volker Quaschning

University of Applied Sciences HTW Berlin, Berlin, Germany

Dr. Jan-Hendrik Schleimer

Humboldt-Universität zu Berlin, Berlin, Germany

Dr. Linus Mattauch

University of Oxford, Oxford, United Kingdom

Prof. Dr. Christian Breyer

LUT University, Lappeenranta, Finland

Dr. Heike Hübener

Wiesbaden, Germany

Prof. Dr. Gottfried Kirchengast

University of Graz, Graz, Austria

Alice Chodura

Berlin, Germany

Dr. Jens Clausen

Borderstep Institute for Innovation and Sustainability, Germany

Prof. Dr. Felix Creutzig

Mercator Research Institute on Global Commons and Climate Change and Technical University Berlin, Berlin, Germany

Marianne Darbi

UFZ - Helmholtz Centre for Environmental Research, Leipzig, Germany

Prof. Dr. Claus-Heinrich Daub

University of Applied Sciences and Arts Northwestern Switzerland,

Windisch, Switzerland

Prof. Dr. Dr. Felix Ekardt

Forschungsstelle Nachhaltigkeit und Klimapolitik, Leipzig/Berlin, and

University of Rostock, Germany
Prof. Dr. Maja Göpel

German Advisory Council on Global Change (WBGU), Berlin, Germany

Dr. Judith N. Hardt

Universität Hamburg, Hamburg, Germany

Dr. Julia Hertin

German Advisory Council on the Environment, Berlin, Germany

Prof. Dr. Thomas Hickler

Senckenberg Biodiversity and Climate Research Center and

Goethe University, Frankfurt am Main, Germany

Dr. Arnulf Köhncke

WWF Germany

Stephan Köster

Alice Salomon Hochschule, Berlin, Germany

Dr. Julia Krohmer

Senckenberg Gesellschaft für Naturforschung, Frankfurt am Main, Germany

Prof. em. Dr. Helga Kromp-Kolb

University of Natural Resources and Life Sciences, Vienna, Austria

Prof. Dr. Reinhold Leinfelder

Freie Universität Berlin, Berlin, Germany

Linda Mederake

Ecologic Institute, Berlin, Germany

Michael Neuhaus

Fridays for Future, Leipzig, Deutschland

Prof. Stefan Rahmstorf, PhD

Potsdam Institute for Climate Impact Research, Potsdam, Germany

Christine Schmidt

Association for Vocational Training Research, Berlin, Germany

Prof. Dr. Christoph Schneider

Humboldt-Universität zu Berlin, Berlin, Germany

Prof. Dr. Gerhard Schneider

University of Applied Sciences and Arts Western Switzerland,

Yverdon-les-Bains, Switzerland

Prof. Dr. Ralf Seppelt

UFZ - Helmholtz Centre for Environmental Research, Leipzig, Germany and Martin-Luther-University Halle-Wittenberg, Halle (Saale), Germany

Prof. Uli Spindler

Technical University of Applied Sciences, Rosenheim, Germany

Dr. Marco Springmann

University of Oxford, Oxford, United Kingdom

Katharina Staab

Berlin, Germany

Prof. Dr. Thomas F. Stocker

University of Bern, Bern, Switzerland

Prof. Dr. Karl Steininger

University of Graz, Graz, Austria

Dr. Eckart von Hirschhausen

Doctors For Future and Allianz Klimawandel und Gesundheit,

Berlin, Germany

Dr. Susanne Winter

WWF Germany

Martin Wittau

German Federal Association for Sustainability, Berlin, Germany

Josef Zens

Helmholtz Centre Potsdam German Research Centre for Geosciences - GFZ,

Potsdam, Germany 